\title{
Corticosteroid status influences the volume of the rat cingulate cortex - a magnetic resonance imaging study
}

\author{
J.J. Cerqueira ${ }^{\text {a,1 }}$, C. Catania ${ }^{\text {b,1 }}$, I. Sotiropoulos ${ }^{\text {b,1 }}$, M. Schubert ${ }^{\text {c,2 }}$, R. Kalisch ${ }^{\text {c,2 }}$, \\ O.F.X. Almeida ${ }^{b}$, D.P. Auer ${ }^{c}$, N. Sousa ${ }^{a, *}$ \\ ${ }^{a}$ Neuroscience Group, Life and Health Sciences Research Institute (ICVS), University of Minho, Campus de Gualtar, 4710-057 Braga, Portugal \\ ${ }^{\mathrm{b}}$ Neuroadaptations Group, Max Planck Institute of Psychiatry, Kraepelinstrasse 2-10, 80804 Munich, Germany \\ ${ }^{\mathrm{c}}$ NeuroImaging Group, Max Planck Institute of Psychiatry, Kraepelinstrasse 2-10, 80804 Munich, Germany
}

Received 20 July 2004; received in revised form 15 November 2004; accepted 11 January 2005

\begin{abstract}
Imbalances in the corticosteroid milieu result in reductions in hippocampal volume in humans and experimental rodents. The functional correlates of these changes include deficits in cognitive performance and regulation of the hypothalamic-pituitaryadrenal axis. Since other limbic structures which are intricately connected with the hippocampal formation, also play an important role in behavioural and neuroendocrine functions, we here used magnetic resonance imaging (MRI) to analyse how two of these areas, the anterior cingulate and retrosplenial cortex, respond to chronic alterations of adrenocortical status: hypocortisolism (induced by adrenalectomy, ADX), normocortisolism (ADX with low-dose corticosterone replacement), and hypercortisolism (ADX with high-dose dexamethasone supplementation). Hypercortisolism was associated with a significant reduction in the volume (absolute and normalized) of the left anterior cingulate gyrus as measured by MRI and confirmed using classical histological methods; a similar trend was observed in the right anterior cingulate region. In contrast, hypercortisolism did not influence the volume of the adjacent retrosplenial cortex. The volumes of the anterior cingulate gyrus and retrosplenial cortex were unaffected by the absence of adrenocortical hormones. These findings are the first to suggest that corticosteroid influences on the structure of the limbic system extend beyond the hippocampal formation, i.e., to fronto-limbic areas also.
\end{abstract}

(C) 2005 Elsevier Ltd. All rights reserved.

Keywords: Corticosterone; Dexamethasone; Retrosplenial cortex; Neuroendocrine regulation; Cognition; Stereology

\section{Introduction}

The prefrontal cortex, including the cingulate cortex, regulates a variety of autonomic functions associated

Abbreviations: ACC, anterior cingulate cortex; ADX, adrenalectomized; ANOVA, analysis of variance; CON, controls; CORT, corticosterone; DEX, dexamethasone; HPA, hypothalamic-pituitaryadrenal axis; MR, magnetic resonance; MRI, magnetic resonance imaging; RSC, retrosplenial cortex; SD, standard deviation.

* Corresponding author. Tel.: +351 253 604806; fax: +351 253 604809 .

E-mail address: njcsousa@ecsaude.uminho.pt (N. Sousa).

${ }^{1}$ These authors contributed equally to this work.

2 These authors contributed equally to this work. with the perception and response to stress in both rats (Devinsky et al., 1995; Frysztak and Neafsey, 1991; Frysztak and Neafsey, 1994; Henke, 1984; Neafsey, 1990; Sullivan and Henke, 1986) and humans (Damasio et al., 1990; Wolf et al., 2002a). Adrenocorticosteroid secretion represents the major endocrine response to stress; besides orchestrating the organism's physical and physiological adjustments to stress, corticosteroids act in the brain to coordinate the behavioural response to stress and can induce changes in hippocampal structure and function. Assimilation of structural observations with large body of results from pharmacological studies has lead to the consensus that corticosteroids influences in the hippocampus are largely dependent 
on the balance between mineralocorticoid receptors (MR) and glucocorticoid receptors (GR) activation (for a review, see Sousa and Almeida, 2002). MR activation appears essential for the survival of existing and newly generated neurons, while GR mediate neuronal atrophy and synaptic loss (Leverenz et al., 1999; Sousa et al., 2000; Vollmann-Honsdorf et al., 1997) or, in extreme cases, cell loss (Sousa et al., 1999). Interestingly, studies in both rodents (Sousa et al., 2000) and humans (Starkman et al., 1999) have demonstrated that hypercortisolemia-induced volumetric reductions are reversible.

Surprisingly, studies on the influence of corticosteroid levels on the structure of other limbic brain regions are sparse despite their implication in the regulation of the hypothalamic-pituitary-adrenal (HPA) axis. Like the hippocampus, the cortex (including the cingulate) expresses corticosteroid receptors (Chao et al., 1989; Herman, 1993). Both the hippocampus and cingulate cortex are activated by stressful stimuli whose interpretation requires reference to previous experience ('processive stressors') and contribute to the neural control of HPA activity (Diorio et al., 1993). Extensive projections from the caudal anterior cingulate cortex (ACC) to the retrosplenial cortex (RSC) suggest that the RSC may also be involved in the control of HPA activity. However, apart from a single report showing that lesions in the RSC lead to significant increases in corticosterone secretion (Suarez and Perassi, 1988), there is a conspicuous lack of information on this issue.

Besides their putative influence on HPA activity, both the ACC and RSC are also implicated in several hippocampus-dependent cognitive functions. For example, the medial prefrontal cortex (which includes the ACC) processes short-term spatial memory in parallel with the hippocampus (Lee and Kesner, 2003), and the RSC is crucial in bridging neocortical and limbic structures involved in allothetic navigation (Whishaw et al., 2001). As variations in the levels of corticosteroids are also known to influence these cognitive processes (McGaugh and Roozendaal, 2002; Oitzl et al., 1997), evaluation of the structure of these extrahippocampal regions following perturbations of the corticosteroid milieu is pertinent.

Evaluation of brain structure (e.g., volume and cell number estimations, synaptic density and dendritic length measurements) is typically conducted postmortem. Direct comparison of post-mortem MR-based hippocampal volumetry in mice at $11.7 \mathrm{~T}$ with stereology on histological slices revealed high correlation between both modalities (Redwine et al., 2003). Recent in vivo MR studies with high field-strength and dedicated head-coils demonstrate the feasibility to perform repeated non-invasive imaging of the rodent brain with submillimeter resolution. Thus, MRI has been employed to study the dynamics of hippocampal lesions in rodent epilepsy models, for example (Bouilleret et al., 2000; John et al., 1996; Roch et al., 2002). Delineation of lesions by MRI report good correlations with histological observations (Allegrini and Sauer, 1992; Ben-Horin et al., 1996). Reliable depiction of limbic structures in the rodent brain has also been demonstrated in vivo at $7 \mathrm{~T}$ (Wolf et al., 2002b). Here, a combined MRI and conventional histological approach was used to address the question of whether chronic alterations of the corticosteroid environment result in structural alterations in the ACC and RSC of the adult rat; detection of changes in these extrahippocampal structures will contribute to our improved understanding of the behavioural and neuroendocrine anomalies associated with disturbances in corticosteroid secretion.

\section{Materials and methods}

\subsection{Animals and treatments}

Male Wistar rats (Charles River, Sulzfeld, Germany), were used in this study. All treatments and in vivo examinations were performed in accordance with the European Communities Council Directives of 24 November 1986 (86/609/EEC) and local regulations on animal welfare. Animals were housed 5-6 per cage under standard environmental conditions (temperature $22{ }^{\circ} \mathrm{C}$; relative humidity $70 \% ; 12 \mathrm{~h}$ light: $12 \mathrm{~h}$ dark cycle [lights on at 6 a.m.]; ad libitum access to food and drinking solution). Treatments were initiated when the animals were 8 weeks of age and were continued over a period of 11 weeks; body weights were recorded twice weekly. The experiment included the following treatment groups:

(i) Controls $(\mathrm{CON})$ were sham-adrenalectomized rats maintained on tap water $(n=5)$.

(ii) Adrenalectomized (ADX) rats (hypocortisolism) were prepared surgically under halothane anesthesia and maintained on $0.9 \%$ saline as drinking solution $(n=5)$.

(iii) Corticosterone-replaced $\mathrm{ADX}$ (ADX + CORT) animals (normo-cortisolism) were ADX as above and received a drinking solution consisting of $7.5 \mu \mathrm{g} / \mathrm{ml}$ corticosterone (CORT) in $0.9 \%$ saline $(n=6)$. CORT (Sigma, Deisenhofen, Germany) was initially dissolved in 2-hydroxy- $\beta$-cyclodextrin (Sigma). Pilot experiments showed that this dose did not activate high-affinity glucocorticoid receptors.

(iv) Dexamethasone-replaced $\mathrm{ADX}(\mathrm{ADX}+\mathrm{DEX})$ rats (hypercortisolism) were ADX as described above and received the prototypic glucocorticoid receptor agonist, dexamethasone (DEX), at a dose 
of $0.25 \mu \mathrm{g} / \mathrm{ml}$ in $0.9 \%$ saline $(n=7)$. Soluble DEX $\left(\right.$ Fortecortin $^{\mathrm{TM}}$ ) was obtained from Merck (Darmstadt, Germany).

\subsection{MRI acquisition}

Animals were transferred to the MRI facility several hours before scanning for adaptation. Animals were anesthetized with halothane, orally intubated and mechanically ventilated at a rate of 50 breaths/min with $1.7 \%$ isoflurane in $40 \% \mathrm{O}_{2} / 60 \% \mathrm{~N}_{2}$. Ventilation was controlled using a Datex AS/3 anesthesia monitor (Datex, Finland). Animals were placed in a custom-built holder with head fixation, an integrated surface head coil (courtesy of M. Neumeir) and a heating pad. Body temperature was monitored with a rectal probe and maintained at $38.0 \pm 0.5^{\circ} \mathrm{C}$. A fibre-optic pulse oximeter (Nonin, USA) was affixed to the left hindpaw to measure arterial $\mathrm{O}_{2}$ saturation and heart rate throughout the experiment.

Imaging was performed on a Bruker $7 \mathrm{~T}$ Avance Biospec 70/30 imager. Using the intrahemispheric cleft as the initial landmark, three mutually orthogonal planes (sagital, axial and coronal) were defined using rapid-acquisition relaxation-enhancement (RARE) scans. This served as a three-dimensional scaffold for the positioning of a package of 20 coronal slices of $0.75 \mathrm{~mm}$ thickness between the most posterior portion of the olfactory bulb and the base of the fourth ventricle. For high resolution imaging a RARE sequence with $\mathrm{TR}=4096 \mathrm{~ms}, \mathrm{TE}_{\mathrm{eff}}=19.4 \mathrm{~ms}$, RARE factor 4 , 6 averages and a $512 \times 384$ matrix $(0.75 \mathrm{~mm}$ slice thickness, $0.1 \mathrm{~mm}$ gap, field of view $3.5 \mathrm{~cm}$ ) was used. Total acquisition time was 39 min $19 \mathrm{~s}$. During reconstruction, images were interpolated to a $512 \times 512$ matrix resulting in a nominal in-plane resolution of $0.068 \times 0.068 \mathrm{~mm}^{2}$.

\subsection{Post-mortem procedures}

Animals were sacrificed by decapitation immediately after removal from the scanner, while they were still anesthetized. Trunk blood was collected for subsequent analysis of serum corticosterone levels by radioimmunoassay (Corticosterone RIA kit, ICN Biochemicals, Costa Mesa, CA). Thymi were excised and maintained on saline-soaked filter papers until weighing.

\subsection{Volumetry}

The ACC was outlined according to the landmarks defined by Wolf et al., 2002b (Fig. 1). Briefly, a line connecting the extreme most dorso-lateral point of the corpus callosum was connected to the most dorsal and medial intra-hemispheric point of the cortex; this line
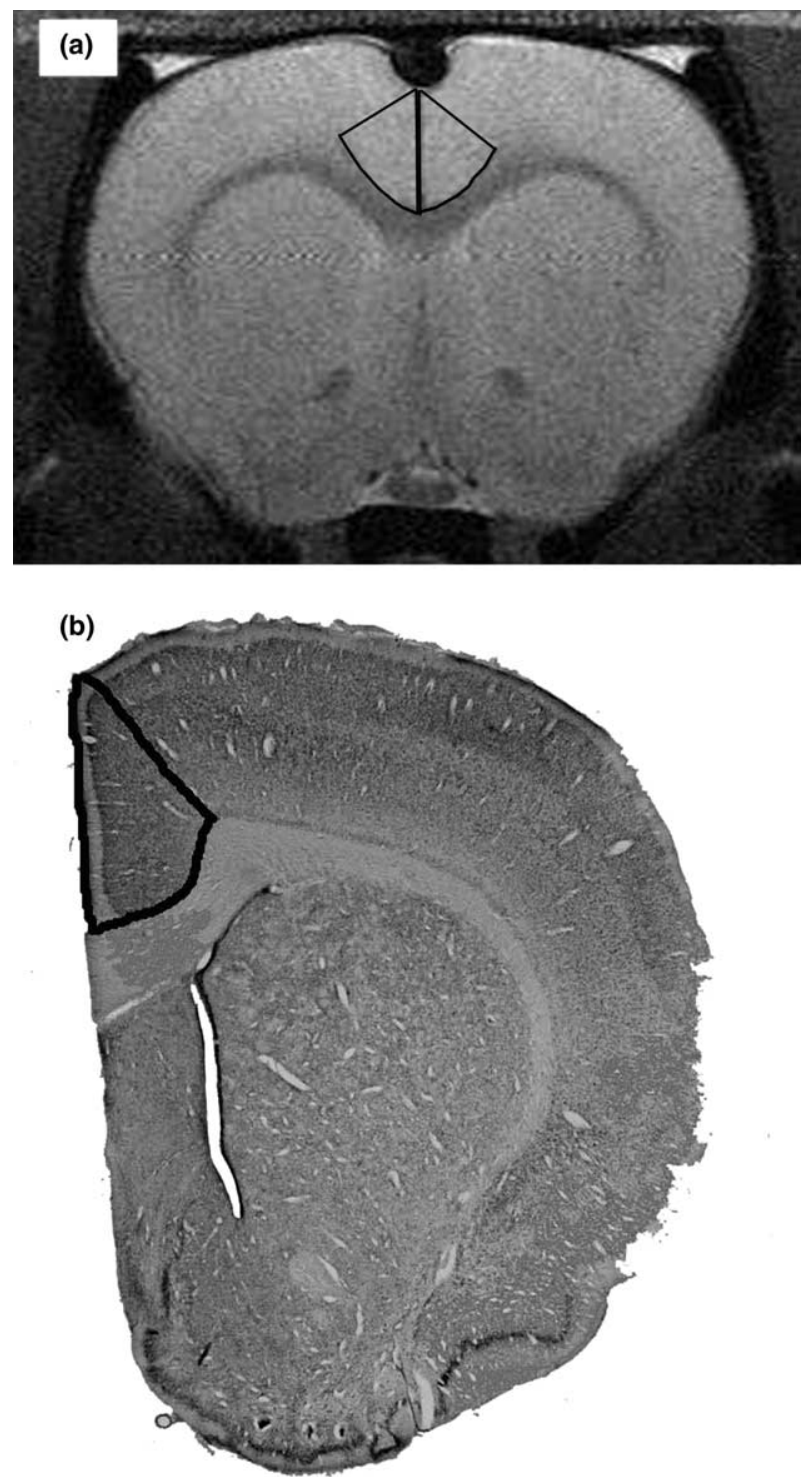

Fig. 1. Landmarks used to delineate the anterior cingulate cortex in MRI scans (a) and histological sections stained with Giemsa (b) are shown. To estimate cross-sectional areas of regions of interest, a systematic set of points was randomly overlaid on an image of the scan/section and the points hitting the area under study were counted (see Section 2).

was continued by the inter-hemispheric line until the intersection of the corpus callosum with the midline and then turned laterally following the corpus callosum to its most dorso-lateral point. The entire cortex within the limits previously defined was measured, starting at the closure of genu of the corpus callosum and terminating at the rostral limit of the hippocampus (see Fig. 2); the chosen profiles for measurement necessarily excluded some of the ACC as its limits are poorly defined in MRI, thus potentially compromising the precision of the volume estimates. Since the retrosplenial granular cortex represents the caudal continuation of the ACC, this region of the brain was measured from the rostral 

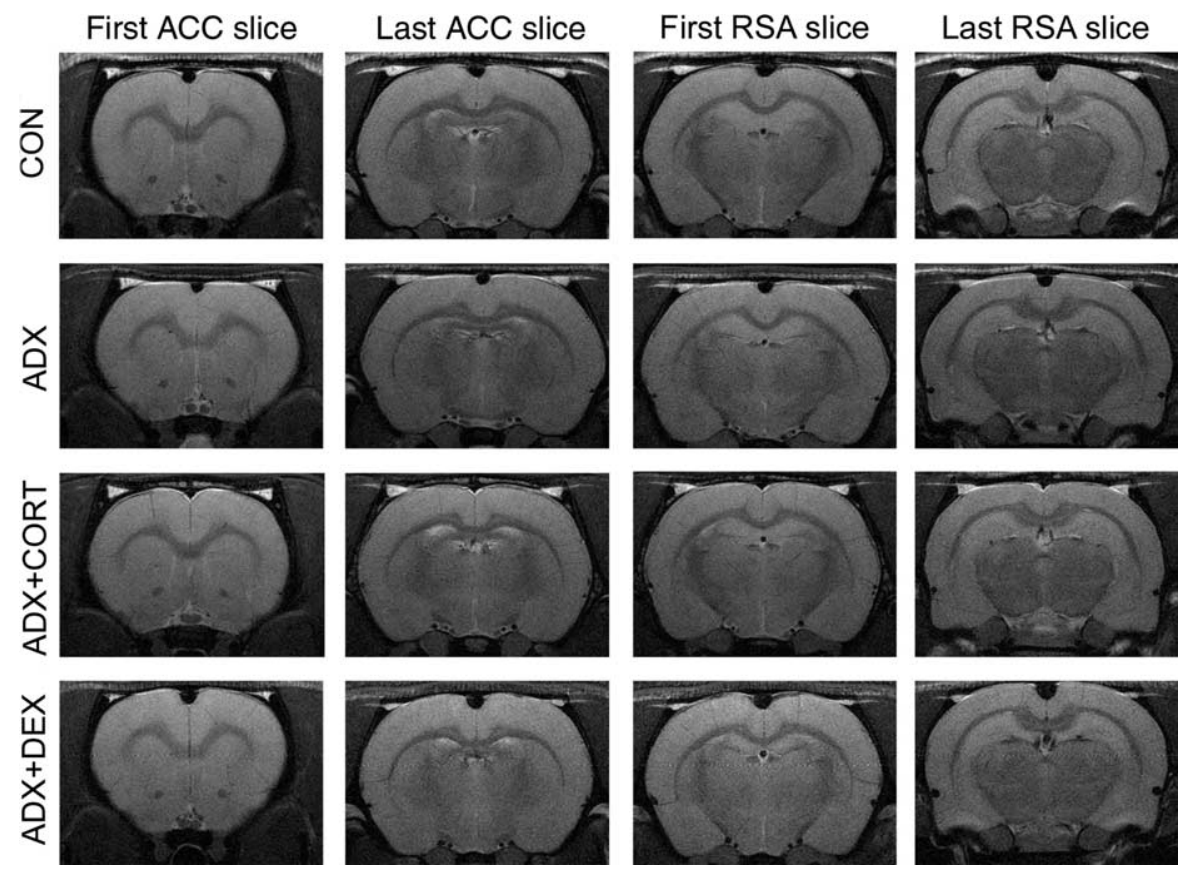

Fig. 2. Examples of the first (rostral) and last (caudal) MR slices used for ACC and RSC volume estimations in one animal of each treatment group. ACC was measured from the closure of the genu of the corpus callosum (first slice where it was closed) to the rostral limit of the hippocampus (last slice before its appearance). RSC was measured from the rostral limit of the hippocampus (first slice where it was present) to the caudal opening of the corpus callosum (last slice before it was opened). See Section 2. CON controls; ADX adrenalectomized rats; ADX + CORT adrenalectomized rats with corticosterone replacement; ADX + DEX adrenalectomized rats with dexamethasone treatment.

limit of the hippocampus until the slice prior to the opening of the corpus callosum (see Fig. 2), using the procedures described above.

The right and left anterior cingulate and retrosplenial areas were manually delineated by one rater (JJC), blinded to treatment status, in consecutive coronal slices containing these regions using StereoInvestigator ${ }^{\mathrm{TM}}$ (MicroBrightField, Williston, VT) software. Intra-rater reliability coefficient was 0.96 . Volumes were calculated by multiplying the area by the inter-slice distance. Determination of hemispheric volume (excluding the olfactory bulb, cerebellum and brain stem) was also performed and cingulate cortex volumes were normalized according to this value.

\subsection{Histological procedures and estimations}

A subset $(n=4)$ of animals from each experimental group was used for histological analysis. The left hemispheres were removed and immediately immersed in isopentane followed by liquid nitrogen. Consecutive $30 \mu \mathrm{m}$ coronal cryosections were stained with Giemsa and coverslipped. Multiple identical linear measurements were taken from fresh sections and in slides following processing; importantly, measures in all three dimensions (along the $x, y$ and $z$ axis) were obtained. The determination of the shrinkage factor $(\mathrm{SFv})$ resulted from the calculation of tissue retraction in every dimension; this procedure was performed in all experimental groups to determine differential tissue shrinkage factors.

The volume of the left ACC was estimated on the basis of the Cavalieri's principle. Briefly, every $4^{\text {th }}$ section was used for the estimates; the cross-sectional area of the ACC was estimated by point counting (final magnification $\times 112$ ) using a test point system in which the interpoint distance, at the tissue level, was $150 \mu \mathrm{m}$. The volume of the left ACC was then calculated from the number of points that fell within the area of interest and the distance between the systematically sampled sections.

\subsection{Statistical analysis}

Results are expressed as means and standard deviations. Effects of treatment were examined by one-way analysis of variance (ANOVA). Post-hoc linear polynomial contrast tests were applied to test whether means differed significantly from each other (pair-wise comparisons). Correlation between volumetric determinations in MRI and histological sections was determined by Pearson's correlation test and BlandAltman agreement analysis (Altman and Bland, 1983). Differences were considered to be significant if $p<0.05$. 


\section{Results}

\subsection{Biological efficacy of hormone manipulations}

The efficacy of the various hormonal manipulations was proven by their effects on body weight as judged by ANOVA $(F=99.7 ; p<0.001)$. Compared to the CON and ADX + CORT-treated groups, ADX resulted in a significant decrease in body weight over the experimental period $(p<0.005)$. ADX + DEX-treated animals showed an even greater loss of body weight as compared to CON $(p<0.001)$ and other treatment groups (vs. $\mathrm{ADX}$ and vs. $\mathrm{ADX}+\mathrm{CORT} p<0.001$ ) (Fig. 3(a)).

ANOVA on ranks showed that all treatments resulted in significant reductions of daytime plasma CORT levels $(F=13.8 ; p=0.003)$ (Fig. 3(b)). ADX and ADX + DEX animals had undetectable levels of CORT. Pair-wise analysis revealed that CORT levels in ADX + CORT animals were significantly higher than in both ADX $(p=0.019)$ and ADX + DEXtreated rats $(p=0.03)$ and significantly lower than those found in CON animals $(p=0.005)$. The elevated CORT levels in adrenal-intact animals reflect the stressful nature of the MRI procedure inasmuch as blood samples were collected immediately after MRI acquisition.

As expected, thymus weight proved to be a better indicator of the corticosteroid status during the entire experimental period. ANOVA revealed a significant effect of treatment on thymus weight at the time of autopsy $(F=6.0 ; p<0.05)$. As compared to controls, the thymus to body weight ratio was significantly increased in ADX animals $(p=0.04)$, and this ratio was significantly decreased in the ADX + DEX group of animals $(p=0.002)$. Attesting to the fact that the CORTreplacement paradigm did not involve occupation of glucocorticoid receptors, ADX + CORT-treated rats did not show any significant reduction in their thymus to body weight ratios (Fig. 3(c)).

\subsection{MR volumetry}

\subsubsection{Hemispheric volumes}

Although there was a slight increase in the volume of both left and right hemispheres in ADX animals and a reduced volume in ADX + DEX-treated animals, ANOVA failed to reveal significant differences $(F=3.1$; $p=0.54$ for right and $F=2.7 ; p=0.74$ for left hemispheric volumes) in this parameter (Fig. 4(a)).

\subsubsection{Anterior cingulate cortex and retrosplenial cortex volumes}

ANOVA indicated a significant effect of treatment on the absolute volume of the left ACC $(F=5.8$;
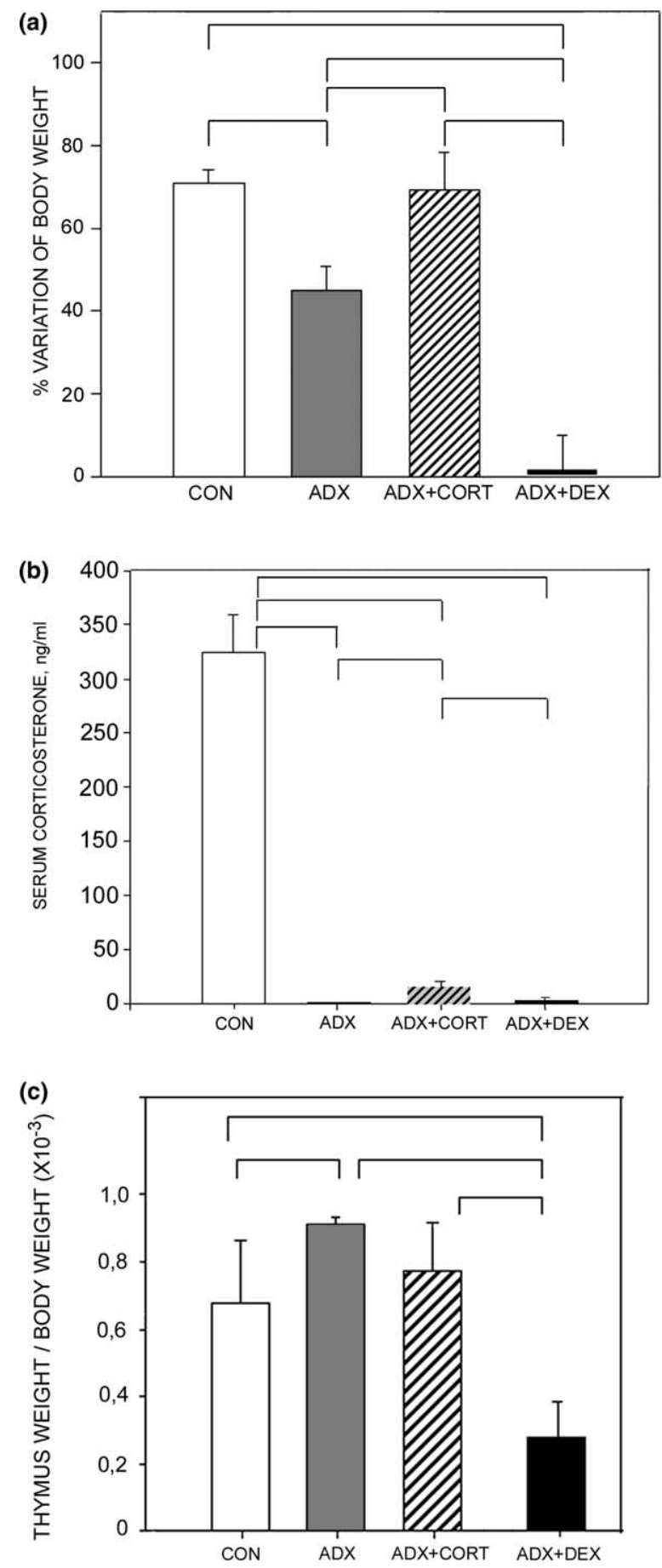

Fig. 3. Effects of hormonal manipulations on body weight (a), serum corticosterone level (b) and thymus/body weight (c). CON controls; ADX adrenalectomized rats; ADX + CORT adrenalectomized rats with corticosterone replacement; ADX + DEX adrenalectomized rats with dexamethasone treatment. Lines indicate significant differences $(p<0.05)$ between experimental groups: (A) CON vs. ADX $p<0.005$; CON vs. ADX + DEX $p<0.001$; ADX vs. ADX + CORT $p<0.005$; ADX vs. ADX $+\operatorname{DEX} p<0.001$; CORT vs. ADX $+\operatorname{DEX} p=0.001$; (B) $\mathrm{CON}$ vs. $\mathrm{ADX} p=0.005$; $\mathrm{CON}$ vs. $\mathrm{ADX}+\mathrm{CORT} p<0.01$; $\mathrm{CON}$ vs. $\mathrm{ADX}+\mathrm{DEX} p<0.01 ; \quad \mathrm{ADX}+\mathrm{CORT}$ vs. $\mathrm{ADX} p<0.02$; $\mathrm{ADX}+\mathrm{CORT}$ vs. $\mathrm{ADX}+\mathrm{DEX} p<0.05$; (C) $\mathrm{CON}$ vs. $\mathrm{ADX}$ $p<0.05$; CON vs. $\mathrm{ADX}+\mathrm{DEX} p=0.002$; $\mathrm{ADX}$ vs. $\mathrm{ADX}+\mathrm{DEX}$ $p<0.001 ; \mathrm{ADX}+\mathrm{CORT}$ vs. ADX $+\operatorname{DEX} p<0.001$. 
(a)

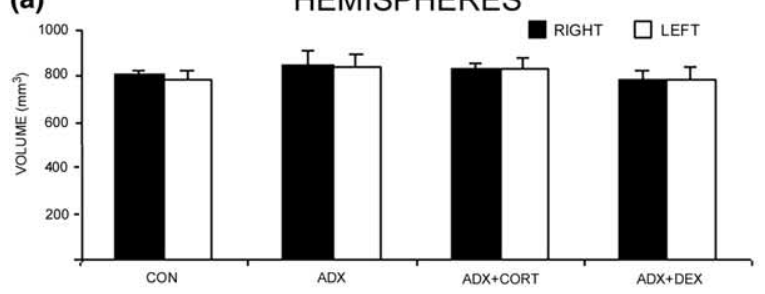

(b)

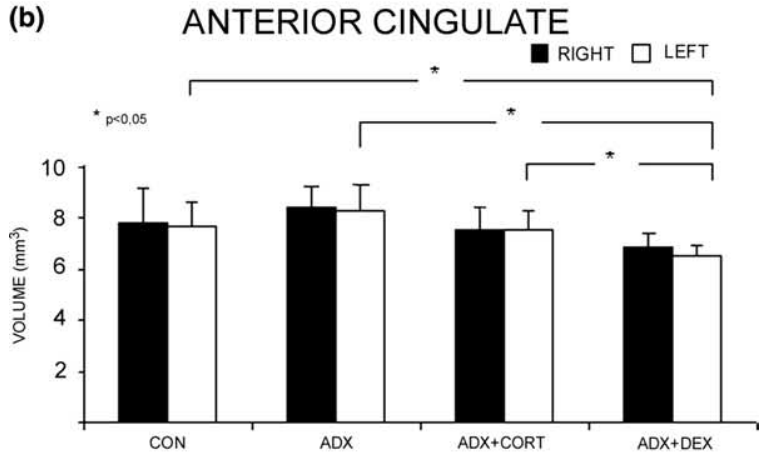

(c) ANTERIOR CINGULATE / HEMISPHERE RATIO

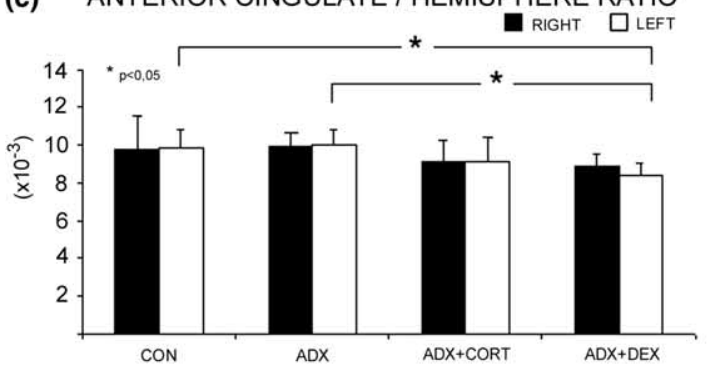

(d)

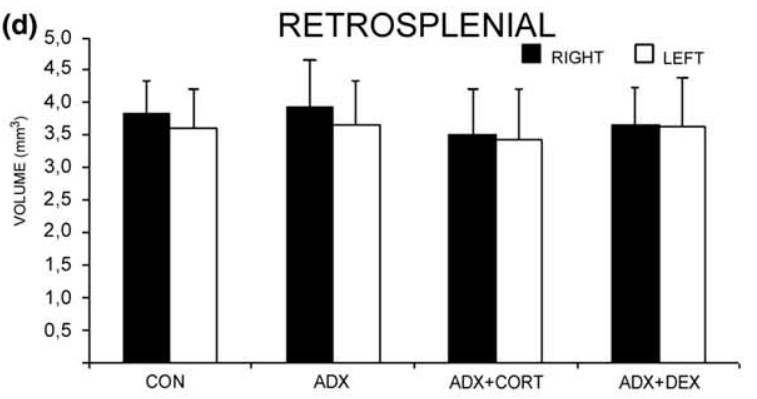

(e) RETROSPLENIAL / HEMISPHERE RATIO

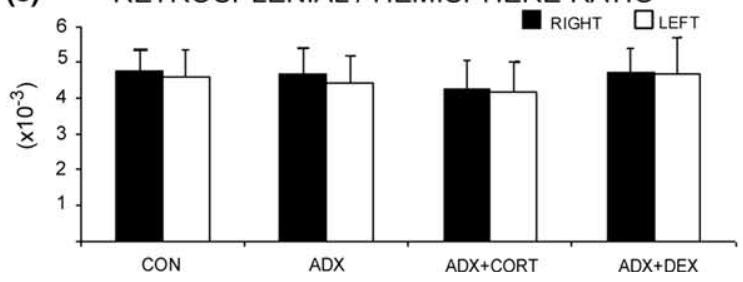

Fig. 4. Volumetric determinations derived from MRI images. CON, controls; ADX adrenalectomized rats; ADX + CORT adrenalectomized rats with corticosterone replacement; ADX + DEX adrenalectomized rats with dexamethasone treatment. (a) Hemispheric volumes. (b) Cingulate cortex volume: $\mathrm{CON}$ vs. $\mathrm{ADX}+\mathrm{DEX} p=0.008$; $\mathrm{ADX}$ vs. $\mathrm{ADX}+\mathrm{DEX} p=0.006 ; \mathrm{ADX}+\mathrm{CORT}$ vs. $\mathrm{ADX}+\mathrm{DEX}$ $p=0.04$. (c) Cingulate cortex volume expressed as a function of hemispheric volumes: $\mathrm{CON}$ vs. $\mathrm{ADX}+\mathrm{DEX} p=0.02$; $\mathrm{ADX}$ vs. $\mathrm{ADX}+\mathrm{DEX} p=0.04$. (d) Retrosplenial cortex volume. (e) Retrosplenial cortex volume expressed as a function of hemispheric volumes. $p<0.005)$. Post-hoc tests revealed a significant reduction on the left ACC in ADX + DEX-treated animals when compared to $\mathrm{CON}(p=0.008), \operatorname{ADX}(p=0.006)$ and $\mathrm{ADX}+\mathrm{CORT}(p=0.04)$-replaced groups. On the contralateral side, there was a similar trend but ANOVA failed to reveal a significant effect of treatment $(F=3.0 ; p=0.06)$ on the volume of the ACC (Fig. 4(b)).

Normalized values (anterior cingulate/hemispheric volume) were also significantly affected by treatment on the left hemisphere $(F=3.4 ; p<0.05)$ but not on the right $(F=1.3 ; p=0.32)$. Comparisons among groups revealed that ADX + DEX-treated animals have a significant reduction of the normalized left ACC volume when compared to controls $(p=0.02)$ and ADX $(p=0.04)$ rats (Fig. 4(c)).

None of the treatments caused significant volumetric alterations in the left or right $\mathrm{RSC}(F=0.49 ; p=0.69$ for right side and $F=0.12 ; p=0.95$ for left side) (Fig. 4(d)).

Normalized values (retrosplenial/hemispheric volume) also failed to reveal significant differences on both hemispheres $(F=0.68 ; p=0.58$ for right and $F=0.46$; $p=0.72$ for left side) (Fig. 4(e)).

In order to test for the occurrence of a shift on the demarcation between the two regions (since we were using external landmarks), we computed and analysed the combined RSA and ACC volumes. ANOVA of these combined volumes revealed a significant effect of treatment on the left hemisphere $(F=4.26 ; p=0.02)$, AD$\mathrm{X}+$ DEX-treated animals having a statistically significant smaller volume $\left(10.2 \pm 0.30 \mathrm{~mm}^{3}\right)$ than both CON $\left(11.6 \pm 0.31 \mathrm{~mm}^{3} ; \quad p=0.006\right)$ and ADX $\left(11.4 \pm 0.19 \mathrm{~mm}^{3} ; p=0.014\right)$ but not ADX + CORTtreated $\left(10.9 \pm 0.3 \mathrm{~mm}^{3} ; p=0.084\right)$ rats. On the right side there was a similar trend $\left(\mathrm{CON} 12.0 \pm 0.48 \mathrm{~mm}^{3}\right.$; ADX $\quad 11.7 \pm 0.30 \mathrm{~mm}^{3} ; \quad$ ADX + CORT $11.1 \pm$ $0.36 \mathrm{~mm}^{3}$; ADX + DEX $10.5 \pm 0.33 \mathrm{~mm}^{3}$ ) but ANOVA failed to reveal a significant effect of treatment $(F=3.198 ; p=0.050)$.

To further elucidate the observed volume reductions, the number of slices measured and a per slice average volume were computed for each region (data shown on Table 1). On the number of slices ANOVA failed to reveal significant differences between groups both for ACC $(F=1.100 ; p=0.376)$ and for RSC $(F=0.305 ; p=0.822)$. According to the whole volume results, analysis of per slice volumes indicated a significant effect of treatment on the left ACC $(F=3.401$; $p=0,042)$, but not on the right ACC $(F=1.678$; $p=0.209)$, the left RSC $(F=0.228 ; p=0.875)$ or the right $\operatorname{RSC}(F=0.093 ; p=0.963)$. Post-hoc analysis confirmed a significant volume reduction on the left ACC in $\mathrm{ADX}+\mathrm{DEX}$-treated rats as compared to $\mathrm{CON}$ $(p=0.02)$ and $\mathrm{ADX} \quad(p=0.015)$ but not $\mathrm{AD}$ $\mathrm{X}+$ CORT-treated $(p=0.106)$ rats. 
Table 1

Detailed analysis of data generated through MR images

\begin{tabular}{lllll}
\hline & CON & ADX & ADX + CORT & ADX + DEX \\
\hline ACC slice number & $5.25 \pm 0.25$ & $5.25 \pm 0.25$ & $5.17 \pm 0.17$ & $4.86 \pm 0.14$ \\
RSC slice number & $3.75 \pm 0.25$ & $3.75 \pm 0.25$ & $3.50 \pm 0.22$ & $3.71 \pm 0.18$ \\
Left ACC per slice volume $^{\mathrm{a}}$ & $1.51 \pm 0.060$ & $1.52 \pm 0.065$ & $1.45 \pm 0.036$ & $1.35 \pm 0.033$ \\
Right ACC per slice volume & $1.55 \pm 0.078$ & $1.52 \pm 0.045$ & $1.46 \pm 0.024$ & $1.41 \pm 0.044$ \\
Left RSC per slice volume & $1.03 \pm 0.018$ & $0.99 \pm 0.021$ & $1.00 \pm 0.029$ & $0.98 \pm 0.068$ \\
Right RSC per slice volume & $0.98 \pm 0.035$ & $0.92 \pm 0.031$ & $0.98 \pm 0.030$ & $0.98 \pm 0.075$ \\
\hline
\end{tabular}

CON, controls; ADX adrenalectomized rats; ADX + CORT adrenalectomized rats with corticosterone replacement; ADX + DEX adrenalectomized rat with dexamethasone treatment. Mean number of MRI slices used for ACC and RSC volume estimations and average per slice volume for each region studied.

${ }^{\mathrm{a}}$ Left ACC per slice volume: CON vs. $\operatorname{ADX}+\operatorname{DEX} p=0.020 ; \operatorname{ADX}$ vs. $\operatorname{ADX}+\operatorname{DEX} p=0.015$.

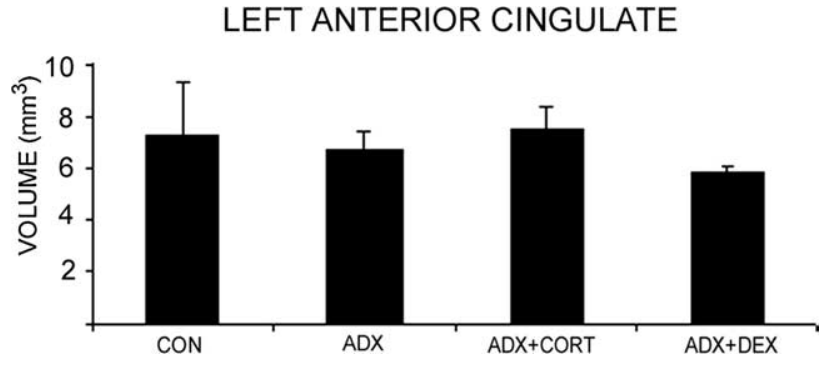

Fig. 5. Volumetric determinations of the left anterior cingulate cortex in histological sections. CON, controls; ADX adrenalectomized rats; ADX + CORT adrenalectomized rats with corticosterone replacement; ADX + DEX adrenalectomized rat with dexamethasone treatment.

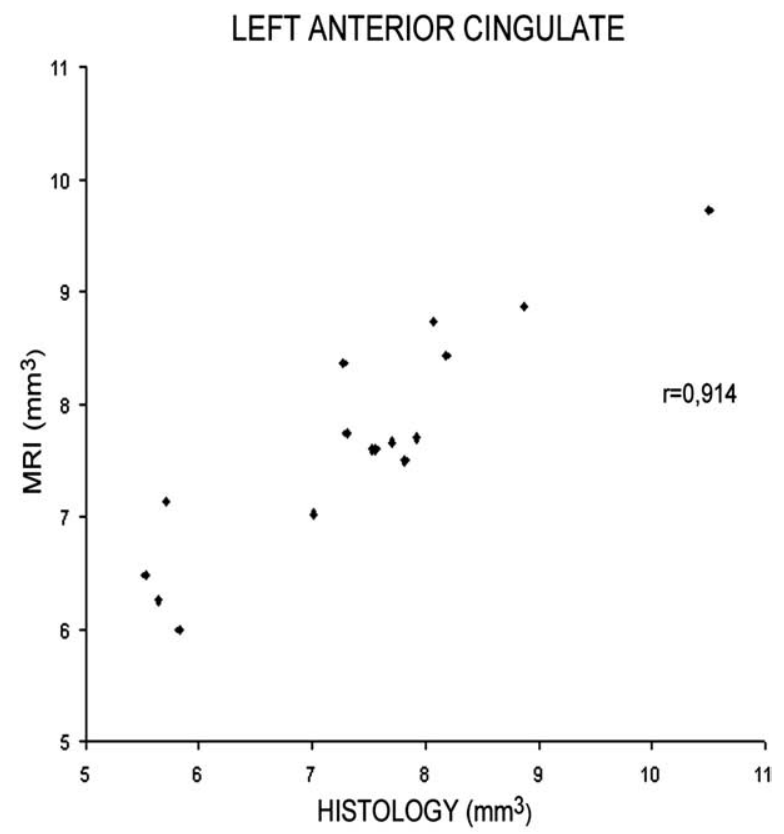

Fig. 6. Correlation between volumetric determinations from MRI images and histological analysis. For each animal, the volume estimated from histological sections is plotted on the $x$-axis and the volumes estimated from MRI against the $y$-axis; $r$ represents Pearson's coefficient of correlation.

\subsection{Histology}

The shrinkage factor was 1.08, 1.17, 1.07 and 1.04, respectively, for controls, ADX, ADX + CORT and ADX + DEX treatment groups. The slight variations found in ADX and ADX + DEX-treated groups are likely to reflect the expected reduced water content in brain tissue of experimental groups (Fig. 5).

Although ANOVA on the histology data did not reach significance $(F=2.83 ; p=0.08)$, a strong correlation was found between the histological and MRI estimations of volumes of the left ACC $(r=0.91$; $p<0.001$ ) (Fig. 6). The mean difference between the two methods of estimation was $-0.28 \pm 0.56 \mathrm{~mm}^{3}$ (Bland-Altman analysis of agreement).

\section{Discussion}

Recent studies demonstrate high correlations between MR volumetry and histology-based morphometry (Redwine et al., 2003); this high degree of agreement between methods is not trivial insofar that it will serve a validation purpose in future applications of MRI for the in vivo evaluation of brain structures on a longitudinal basis, i.e., repeated measures in the same individual. In addition, recent advances in the application of contrast agents, ranging from selectively staining hippocampal substructures with Mn (Watanabe et al., 2002) to cellspecific labeling with ferrous derivatives compounds (Bulte et al., 2003), promise more detailed in vivo analysis in the future.

Despite the limited number of animals in each experimental group, the results of the present study revealed a significant correlation between ACC volumes obtained by MRI assessment vs. conventional histology-based analysis. It should be noted that the ability to precisely outline the cingulate gyrus (except for its most rostral portion) contributes importantly to this significant correlation. It is pertinent to note that in parallel study of the hippocampal formation, using the same 
experimental paradigm, we also observed a high correlation between histology-based morphology and MR volumetry (Schubert et al., 2004).

That corticosteroids influence hippocampaldependent learning and memory processes is now well established (Arbel et al., 1995; Endo et al., 1996; Sousa and Almeida, 2002). Importantly, most studies on the neuromorphological effects of altered corticosteroid status have, to date, focused on the hippocampal formation because of this area's high concentration of corticosteroid receptors, its key role in memory and learning processes and its involvement in the control of the HPA axis. As an example, it has been shown that depressed patients, who have impaired glucocorticoid negative feedback of the HPA axis, also have decreased hippocampal volumes (see for a review Campbell et al., 2004). However, the full spectrum of effects of corticosteroid imbalance on the structure and function of other brain regions, including areas displaying significant levels of corticosteroid receptors, remains under-appreciated. This study redressed this deficit in knowledge with respect to the ACC and RSC. Both these structures express (predominantly) glucocorticoid receptors (Chao et al., 1989; Diorio et al., 1993), and have been implicated in cognitive processes similar to those in which hippocampal involvement has been demonstrated (see Jackson et al., 1998; Kesner et al., 1996; Lee and Kesner, 2003); further, these regions are also thought to contribute to the control of HPA axis activity (Diorio et al., 1993).

Decreases in the volume of the hippocampal formation, primarily due to changes in the volume of the dentate gyrus, have been previously reported after ADX in rats (Sloviter et al., 1989). Interestingly, the opposite hormonal condition (i.e., hypercortisolism) also results in significant decreases in the volumes of several layers of the hippocampal formation (Sousa et al., 1998). The present study in which the effects of hypo- and hyper-cortisolism on the volumes of the cingulate (anterior and retrosplenial) cortex were evaluated are, to the best of our knowledge, the first of their kind. No detrimental effects of ADX could be observed on ACC or RSC volumes. In contrast, a significant reduction was found in the volume of the ACC in ADX animals supplemented with dexamethasone (ADX + DEX). While this difference was only statistically significant in the left hemisphere, a similar reduction was also noted in the contralateral hemisphere. Remarkably, and demonstrating the selective vulnerability of the ACC to the induced hypercortisolismic state, no volumetric differences were found in the adjacent RSC. These findings prompted us to analyse ACC volumes in histological sections in order to confirm the volume estimates provided by MRI scanning; indeed, we observed a strong correlation between the MRI and histological data.
The volumetric differences found in the ACC of dexamethasone-treated animals most likely depend on the relative abundance of the two corticosteroid receptors in this region: while the ACC expresses a high level of glucocorticoid receptors, its complement of mineralocorticoid receptors is low (Chao et al., 1989). It therefore appears that the relative abundance of each corticosteroid receptor subtype, rather than its absence per se, may render the ACC vulnerable to the excess of circulating corticosteroids. Interestingly, although the RSC shows a pattern of corticosteroid receptor distribution similar to that found in the ACC, dexamethasoneinduced hypercortisolism did not produce marked changes in RSC volume. This result resembles the observation that pyramidal neurons in the hippocampus are not subject to ADX, corticosteroid and stress-induced cell death despite their expression of both mineralocorticoid and glucocorticoid receptors (Hassan et al., 1999; Sloviter et al., 1989). Since virtually all neurons express corticosteroid receptors, the present differential responses of the ACC and RSC (as well as of the various hippocampal cell types) raise the interesting question of what might be the unique properties of particular cell types (e.g., anterior cingulate cells) that render them more vulnerable to corticosteroids.

Extensive and reciprocal connections between the hippocampus and prefrontal cortex have been previously described. As previously mentioned, the ACC and possibly the RSC are implicated in the control of the HPA axis (Diorio et al., 1993; Feldman et al., 1995; Mizoguchi et al., 2003) and, therefore, may also be players in brain dysfunctions associated with dysregulation of this axis (e.g., depression, anxiety, posttraumatic stress disorder) (Hamner et al., 1999), as well as in disorders of cognition (Lupien et al., 1999, 2002; Monk and Nelson, 2002; Newcomer et al., 1998; Porter et al., 2002). Importantly, chronic stress, which results in activation of glucocorticoid receptors, is known to induce dysfunction of both the hippocampus (McEwen, 1999) and prefrontal cortex (Mizoguchi et al., 2000).

In conclusion, the present results demonstrate that manipulations of the corticosteroid milieu affect the structure of the limbic system beyond the hippocampal formation, including the ACC. Curiously, despite the intimate interconnectivity between the anterior and posterior divisions of the cingulate cortex, the presentlyobserved differential vulnerability of these structures to dexamethasone exposure is intriguing and warrants further enquiry with respect to the potential mechanisms underlying this difference.

\section{References}

Allegrini PR, Sauer D. Application of magnetic resonance imaging to the measurement of neurodegeneration in rat brain: MRI data 
correlate strongly with histology and enzymatic analysis. Magnetic Resonance Imaging 1992;10:773-8.

Altman DG, Bland JM. Measurement in medicine: the analysis of method comparison studies. Statistician 1983;32:307-17.

Arbel I, Kadar T, Silbermann M, Levy A. The effects of long-term corticosterone administration on hippocampal morphology and cognitive performance of middle-aged rats. Brain Research 1995;657:227-35.

Ben-Horin N, Hazvi S, Bendel P, Schul R. The ontogeny of a neurotoxic lesion in rat brain revealed by combined MRI and histology. Brain Research 1996;718:97-104.

Bouilleret V, Nehlig A, Marescaux C, Namer IJ. Magnetic resonance imaging follow-up of progressive hippocampal changes in a mouse model of mesial temporal lobe epilepsy. Epilepsia 2000;41:642-50.

Bulte JW, Ben-Hur T, Miller BR, Mizrachi-Kol R, Einstein O, Reinhartz E, Zywicke HA, Douglas T, Frank JA. MR microscopy of magnetically labeled neurospheres transplanted into the Lewis EAE rat brain. Magnetic Resonance in Medicine 2003;50:201-5.

Campbell S, Marriott M, Nahmias C, MacQueen GM. Lower hippocampal volume in patients suffering from depression: a meta-analysis. American Journal of Psychiatry 2004;161:598-607.

Chao HM, Choo PH, McEwen BS. Glucocorticoid and mineralocorticoid receptor mRNA expression in rat brain. Neuroendocrinology 1989;50:365-71.

Damasio AR, Tranel D, Damasio H. Individuals with sociopathic behavior caused by frontal damage fail to respond autonomically to social stimuli. Behavioural Brain Research 1990;41:81-94.

Devinsky O, Morrell MJ, Vogt BA. Contributions of anterior cingulate cortex to behaviour. Brain 1995;118:279-306.

Diorio D, Viau V, Meaney MJ. The role of the medial prefrontal cortex (cingulate gyrus) in the regulation of hypothalamicpituitary-adrenal responses to stress. Journal of Neuroscience 1993;13:3839-47.

Endo Y, Nishimura JI, Kimura F. Impairment of maze learning in rats following long-term glucocorticoid treatments. Neuroscience Letters 1996;203:199-202.

Feldman S, Conforti N, Weidenfeld J. Limbic pathways and hypothalamic neurotransmitters mediating adrenocortical responses to neural stimuli. Neuroscience and Biobehavioural Reviews 1995;19:235-40.

Frysztak RJ, Neafsey EJ. The effect of medial frontal cortex lesions on respiration, "freezing" and ultrasonic vocalizations during conditioned emotional responses in the rat. Cerebral Cortex 1991;1:418-25.

Frysztak RJ, Neafsey EJ. The effect of medial frontal cortex lesions on cardiovascular conditioned emotional responses in the rat. Brain Research 1994;643:181-93.

Hamner MB, Lorberbaum JP, George MS. Potential role of the anterior cingulate cortex in PTSD: review and hypothesis. Depression and Anxiety 1999;9:1-14.

Hassan AH, Patchev VK, von Rosenstiel P, Holsboer F, Almeida OF. Plasticity of hippocampal corticosteroid receptors during aging in the rat. FASEB Journal 1999;13:115-22.

Henke PG. The anterior cingulate cortex and stress: effects of chlordiazepoxide on unit-activity and stimulation induced gastric pathology in rats. International Journal of Psychophysiology 1984;2:23-32.

Herman JP. Regulation of adrenocorticosteroid receptor mRNA expression in the central nervous system. Cellular and Molecular Neurobiology 1993;13:349-72.

Jackson PA, Kesner RP, Amann K. Memory for duration: role of hippocampus and medial prefrontal cortex. Neurobiology of Learning and Memory 1998;70:328-48.

John J, Govindaraju V, Raghunathan P, Kumar VM. Magnetic resonance imaging of temporal changes of neurotoxic lesion in the rat. Brain Research Bulletin 1996;40:273-7.
Kesner RP, Hunt ME, Williams JM, Long JM. Prefrontal cortex and working memory for spatial response, spatial location, and visual object information in the rat. Cerebral Cortex 1996;6:311-8.

Lee I, Kesner RP. Time-dependent relationship between the dorsal hippocampus and the prefrontal cortex in spatial memory. Journal of Neuroscience 2003;23:1517-23.

Leverenz JB, Wilkinson CW, Wamble M, Corbin S, Grabber JE, Raskind MA, Peskind ER. Effect of chronic high-dose exogenous cortisol on hippocampal neuronal number in aged nonhuman primates. Journal of Neuroscience 1999;19:2356-61.

Lupien SJ, Gillin CJ, Hauger RL. Working memory is more sensitive than declarative memory to the acute effects of corticosteroids: a dose-response study in humans. Behavioural Neuroscience 1999;113:420-30.

Lupien SJ, Wilkinson CW, Briere S, Menard C, Ng Ying Kin NM, Nair NP. The modulatory effects of corticosteroids on cognition: studies in young human populations. Psychoneuroendocrinology 2002;27:401-16.

McEwen BS. Stress and hippocampal plasticity. Annual Review of Neuroscience 1999;22:105-22.

McGaugh JL, Roozendaal B. Role of adrenal stress hormones in forming lasting memories in the brain. Current Opinion in Neurobiology 2002;12:205-10.

Mizoguchi K, Yuzurihara M, Ishige A, Sasaki H, Chui DH, Tabira T. Chronic stress induces impairment of spatial working memory because of prefrontal dopaminergic dysfunction. Journal of Neuroscience 2000;20:1568-74.

Mizoguchi K, Ishige A, Aburada M, Tabira T. Chronic stress attenuates glucocorticoid negative feedback: involvement of the prefrontal cortex and hippocampus. Neuroscience 2003;119:887-97.

Monk CS, Nelson CA. The effects of hydrocortisone on cognitive and neural function: a behavioural and event-related potential investigation. Neuropsychopharmacology 2002;26:505-19.

Neafsey EJ. Prefrontal cortical control of the autonomic nervous system: anatomical and physiological observations. Progress in Brain Research 1990;85:147-65.

Newcomer JW, Craft S, Askins K, Hershey T, Bardgett ME, Csernansky JG, Gagliardi AE, Vogler G. Glucocorticoid interactions with memory function in schizophrenia. Psychoneuroendocrinology 1998;23:65-72.

Oitzl MS, van Haarst AD, de Kloet ER. Behavioural and neuroendocrine responses controlled by the concerted action of central mineralocorticoid (MRS) and glucocorticoid receptors (GRS). Psychoneuroendocrinology 1997;22(Suppl. 1):S87-93.

Porter RJ, Barnett NA, Idey A, McGuckin EA, O'Brien JT. Effects of hydrocortisone administration on cognitive function in the elderly. Journal of Psychopharmacology 2002;16:65-71.

Redwine JM, Kosofsky B, Jacobs RE, Games D, Reilly JF, Morrison JH, Young WG, Bloom FE. Dentate gyrus volume is reduced before onset of plaque formation in PDAPP mice: a magnetic resonance microscopy and stereologic analysis. Proceedings of the National Academy of Sciences of the United States of America 2003;100:1381-6.

Roch C, Leroy C, Nehlig A, Namer IJ. Magnetic resonance imaging in the study of the lithium-pilocarpine model of temporal lobe epilepsy in adult rats. Epilepsia 2002;43:325-35.

Schubert M, Kalisch R, Sotiropoulos I, Catania C, Almeida OFX, Sousa $\mathrm{N}$, et al. In vivo assessment of hippocampal volume and neurochemical changes in rats with altered corticosteroid milieu. Abstracts of the 12th scientific meeting of the international society for magnetic resonance in medicine; 2004.

Sloviter RS, Valiquette G, Abrams GM, Ronk EC, Sollas AL, Paul LA, Neubort S. Selective loss of hippocampal granule cells in the mature rat brain after adrenalectomy. Science 1989;243:535-8.

Sousa N, Madeira MD, Paula-Barbosa MM. Effects of corticosterone treatment and rehabilitation on the hippocampal formation of 
neonatal and adult rats. An unbiased stereological study. Brain Research 2002b;794:199-210.

Sousa N, Paula-Barbosa MM, Almeida OF. Ligand and subfield specificity of corticoid-induced neuronal loss in the rat hippocampal formation. Neuroscience 1999;89:1079-87.

Sousa N, Lukoyanov NV, Madeira MD, Almeida OF, Paula-Barbosa MM. Reorganization of the morphology of hippocampal neurites and synapses after stress-induced damage correlates with behavioural improvement. Neuroscience 2000;97:253-66.

Sousa N, Almeida OF. Corticosteroids: sculptors of the hippocampal formation. Reviews in the Neurosciences 2002;13:59-84.

Starkman MN, Giordani B, Gebarski SS, Berent S, Schork MA, Schteingart DE. Decrease in cortisol reverses human hippocampal atrophy following treatment of Cushing's disease. Biological Psychiatry 1999;46:1595-602.

Suarez M, Perassi NI. Plasma corticosterone responses to lesions and stimulations of the limbic thalami nuclei, medial mammillary nucleus and cingulate cortex. Archives Internationales de Physiologie et de Biochimie 1988;96:69-74.

Sullivan RM, Henke PG. The anterior midline cortex and adaptation to stress ulcers in rats. Brain Research Bulletin 1986;17:493-6.
Vollmann-Honsdorf GK, Flugge G, Fuchs E. Chronic psychosocial stress does not affect the number of pyramidal neurons in tree shrew hippocampus. Neuroscience Letters 1997;233:121-4.

Watanabe $\mathrm{T}$, Natt $\mathrm{O}$, Boretius $\mathrm{S}$, Frahm $\mathrm{J}$, Michaelis $\mathrm{T}$. In vivo 3D MRI staining of mouse brain after subcutaneous application of $\mathrm{MnCl}_{2}$. Magnetic Resonance in Medicine 2002;48:852-9.

Whishaw IQ, Maaswinkel H, Gonzalez CL, Kolb B. Deficits in allothetic and idiothetic spatial behavior in rats with posterior cingulate cortex lesions. Behavioural Brain Research 2001;118:67-76.

Wolf OT, Convit A, de Leon MJ, Caraos C, Quadri SF. Basal hypothalamo-pituitary-adrenal axis activity and corticotropin feedback in young and older men: relationships to magnetic resonance imaging-derived hippocampus and cingulate gyrus volumes. Neuroendocrinology 2002a;75:241-9.

Wolf OT, Dyakin V, Vadasz C, de Leon MJ, McEwen BS, Bulloch K. Volumetric measurement of the hippocampus, the anterior cingulate cortex, and the retrosplenial granular cortex of the rat using structural MRI. Brain Research Protocols 2002b;10:41-6. 